\title{
Method of floc classification after the coagulation process
}

\author{
Ewelina Łukasiewicz ${ }^{1, *}$, and Mariusz Rząsa ${ }^{1}$ \\ ${ }^{1}$ Opole University of Technology, Department of Thermal Engineering and Industrial Facilities, \\ 5 Mikołajczyka Street, 45-271 Opole, Poland
}

\begin{abstract}
Coagulation is one of the processes of water and wastewater treatment. During this process, flocs are formed and are characterized by different shapes, structures and dimensions. Due to this fact, removing of these particles may produce some problems for researchers because the sedimentation way of this particles is disturbed. In this paper, the method of flocs classification is presented where coefficients $\mathrm{k}_{1}, \mathrm{k}_{2}$ and $\mathrm{k}_{3}$ were calculated. An appropriate value of coefficient $\mathrm{k}$ enables the classification of the considered particle to a specific group. The idea of the classification method consists in calculating surface areas for a particle image registered using a microscope. After that, the images are analyzed (computer image processing). The analyzed images will be used for future studies, in which the settling phenomenon will be described.
\end{abstract}

\section{Introduction}

Safe drinking water is essential to the health and welfare of the community, and water from all sources must undergo some form of purification before consumption. A very important step in the process of purifying is a coagulation and flocculation process, which is widely used due to its simplicity and cost-effectiveness. Regardless of the nature of the treated sample (e.g. various types of water or wastewater) and the overall applied treatment scheme, coagulation-flocculation is usually included, either as a pre-, or as post-treatment step [1-3]. Properly carried coagulation not only provides a high degree of removal of colloidal suspensions and settling, but is alsoassociated with these other pollutants, including micropollutants. The impact of coagulation involves an effective reduction of turbidity, color intensity, indicating the organic impurities in these precursors of disinfection by-products and chemical oxidation, and the content of a number of micropollutants, mainly present in the purified water in the form of sparingly soluble form or associates with solid particles. The suspended particles vary considerably in source, composition charge, particle size, shape, and density. Correct application of coagulation and flocculation processes and selection of the coagulants depend upon the understanding of the interaction between these factors $[4,5,6]$. In practice, the coagulation is carried out by coagulants, where the hydrolysis products cause electrolyte coagulation and the coagulation by the colloid with an opposite sign. Coagulation occurs in two phases: in the first - after

*Corresponding author: e.lukasiewicz@po.opole.p1 
the addition of a coagulant, lasting seconds - chemical and physical reactions take place leading to the destabilization of colloidal particles, and in the second phase - lasting much longer - flocculation - the flocs are created as a result of transport and collisions of destabilized particles (Fig. 1). Flocs can be efficiently removed by the process of sedimentation/flotation and filtration [7,8]. After mixing, the processed water is directed to clarifiers. Settling tanks are devices in which a process that removes the suspension from purified water occurs. They are used to retain the settleable suspension easily, either natural or produced by the coagulation process and chemical precipitation of sparingly soluble compounds. Contact settlers with a suspended sediment are used to treat water by coagulation and are usually called clarifiers $[9,10]$. Due to the fact that the drag coefficient depends on both the shape and density of particles, there are some difficulties in determining it. The determination of this coefficient is significantly hindered due to different forms of flocs as a result of the coagulation process. Sediment structures formed during this process are diverse in terms of their shapes and degree of density [11, 12]. Taking the above into consideration, it can be observed that the modelling of this process is extremely difficult. It is possible to group flocs, taking their characteristics into account, and then formulate general equations describing the movement of particles belonging to a given group.

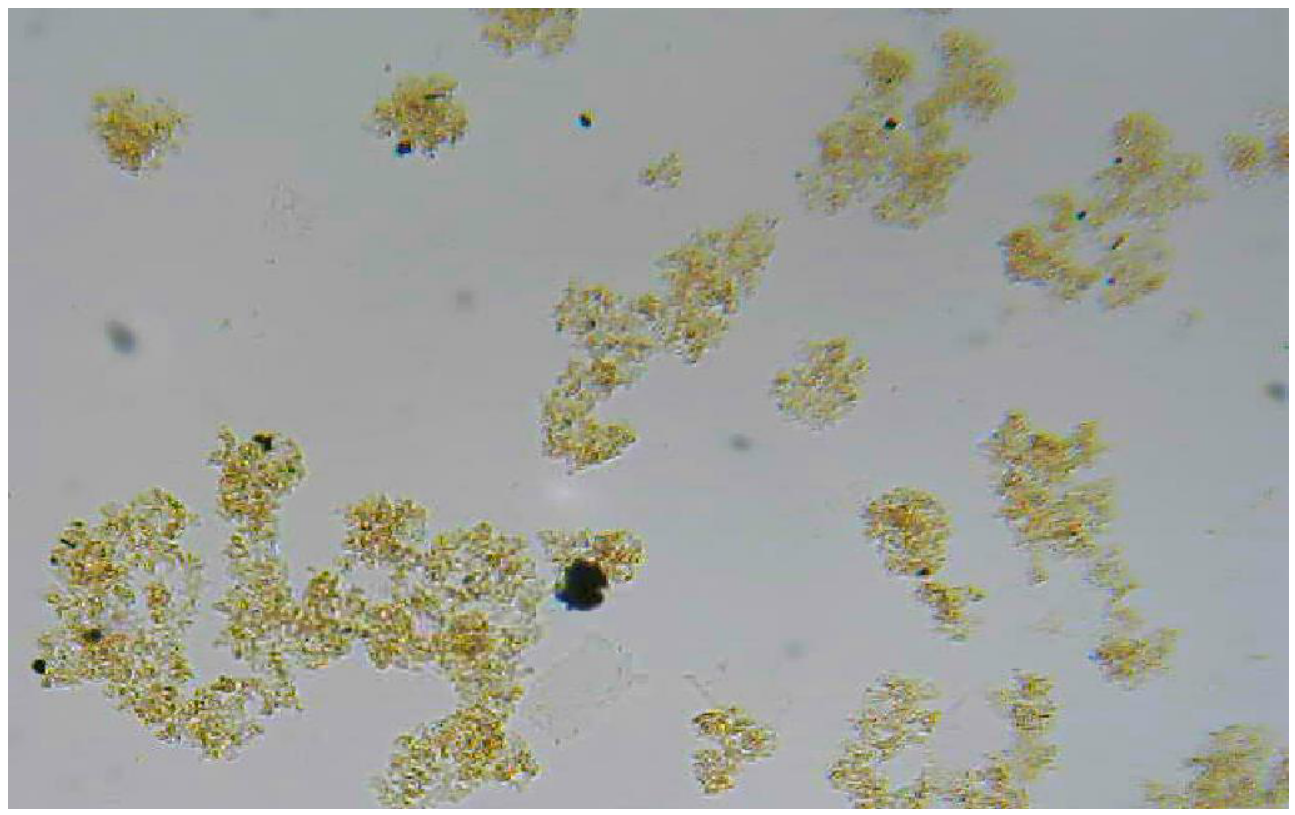

Fig. 1. Post-coagulation suspension.

\section{Classification}

This paper presents the classification of flocs. The classification concerns particle distribution, taking into account their movement during the settling process. Due to technological reasons, the settling time of the sediment is the most important parameter. Therefore, the classification proposed by the authors mainly focuses on the separation of groups of flocs according to their characteristic features affecting the settling velocity.

In order to form a suspension, model water based on distilled water was prepared. $24 \mathrm{~g}$ of sodium chloride, $12 \mathrm{~g}$ of sodium sulfate, and $0.05 \mathrm{~g}$ of iron sulfate were dissolved in 1 liter of water. $60 \mathrm{~g}$ of aluminum hydroxychloride per 1 cubic meter were used as 
a coagulant. After the coagulation process, suspension samples were taken and examined under a microscope.

While examining the microscope images, the authors decided to classify individual particles statistically. Flocs were divided into three characteristic groups. The classification takes into account the structure of the floc in terms of mass concentration (presence of macropores) and its shape. Flocs were divided into: compact particles, particles having a distinct mass concentration nucleus around which sediment crystallization networks are formed (flocks without mass concentration), and particles having a porous sponge structure (porous conglomerates).

Based on the floc analysis, three separate types of particles characterised by a different settling way were distinguished (Fig. 2). Compact particles are characterised a homogeneous structure. Their shape is similar to a sphere filled with the same microporous structure. Micropores are specific features of a post-coagulation material. Particles of this type settle in a straight line with a slight zigzag motion. Due to their small size and mass, their movement is repeatedly disrupted by fluid movement triggered by the movement of other particles, considerably higher in size, in their vicinity. The second group of particles (star-shaped particles) has a distinct mass concentration nucleus around which sediment crystallisation networks are formed (flocs without mass concentration) (Fig.3). Protruding arms cause the particle to move in a circular motion around its own axis while settling. The circular motion causes the particle, whose direction is constantly changing, to be influenced by the Magnus effect. This effect causes the particles of this type to settle in a spiral motion. Over time, star - shaped particles connect to create bigger agglomerates. In case of big agglomerates, the circular motion around their own axis gradually disappears, and particles of this type settle in a straight line. They are characterised by considerably big macropores, through which fluid may flow during their motion. This type of structure is similar to fluid flow through a porous medium [Fig. 4].

a)

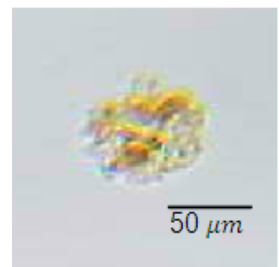

b)

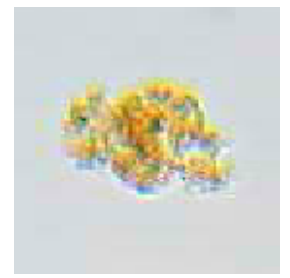

c)

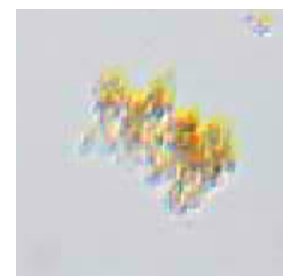

Fig. 2. Compact structure of flocs.

Therefore, the flocs presented above (Fig. 2.) are flocs with a compact structure containing a large number of pores. These particles are characterized by the compact structure and relatively regular shape of a floc. Pores occurring in it are a natural feature of this type of suspensions. Settling of such particles can be described using the Stokes' law [7]. Due to various shapes, it is necessary to determine an appropriate drag coefficient experimentally.

The star-shaped particles have a nucleus with concentrated mass surrounded by a number of sediment crystallization arms (Fig. 3.). In case of such particles, the weight of a nucleus mainly affects the settling velocity. However, irregular crystallization arms change the direction in which particles settle. A non-linear trajectory significantly extends both settling path and time. The mathematical description of such particles requires much more complex mathematical models.

The third group includes porous conglomerates consisting of the types of particles present in a given suspension, combined into clusters. Their structure resembles a sponge, they are very unstable and they can easily change their shape while settling. 
Figure 4 presents the mass that is not concentrated in one place. The structure developed a porous structure (like a sponge). In addition, it was observed that the porous structure of flocs enables them to combine into larger conglomerates. According to the phenomenon of settling conglomerates, this type of structure is classified as a separate group in the classification of particles. The occurrence of a distinct porous structure will result in an additional flow of liquid through open pores.

a)

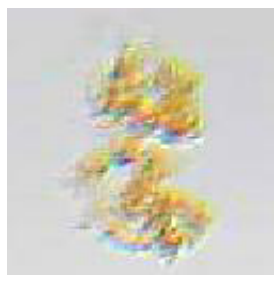

b)

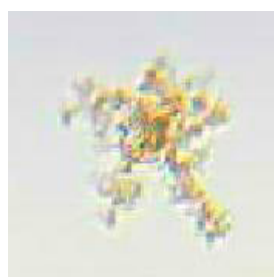

c)

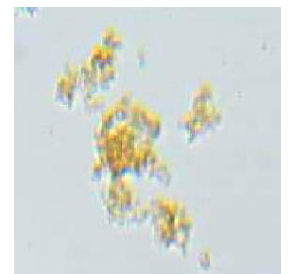

Fig. 3. Flocks without mass concentration.

a)

b)

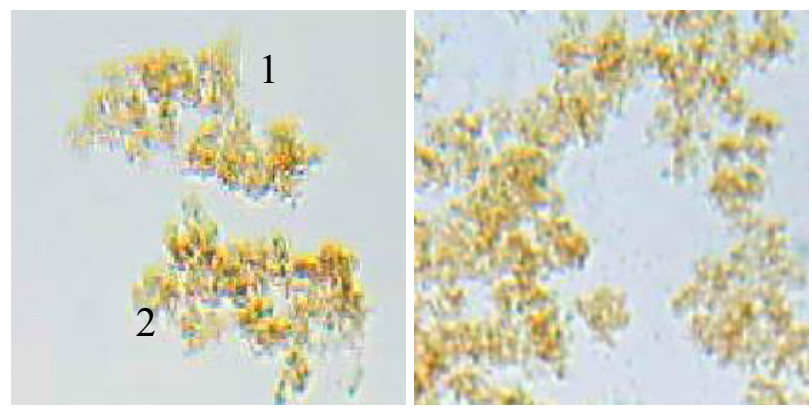

Fig. 4. Porous conglomerates.

\section{Idea of the method of particle classification}

The presented method of classification of post-coagulation particles is based on the process of image analysis (computer image processing). The idea of the classification method consists in calculating surface areas for a image registered using the microscope. Due to a great amount of data (microscope images), it is necessary to apply an automated classification of the flocs of the suspension. In order to carry out such a classification, reference levels in the form of numeral parameters are vital.

The microscope image of a particle (Fig. 5a) is subjected to a binearization process (Fig. 5b). Subsequently, for this image the surface area is calculated for particle $A_{1}$. In the second stage, the computer image analysis is carried out to close all open spaces (Fig. 5c). On this basis, surface area $A_{2}$ is calculated. A similar operation can be performed on the image of Figure $5 \mathrm{~b}$ to make all surfaces closed (Fig. 5d). This operation enables the determination of surface $A_{3}$ constituting the surface of the particles inside the outline.

The calculation of areas $A_{1}, A_{2}$ and $A_{3}$ is the basis for determining the classification criterion. Based on the relationships of selected surface areas, a characteristic factor $\mathrm{k}_{1}, \mathrm{k}_{2}$, and $\mathrm{k}_{3}$ is determined.

$$
k_{1}=\frac{2 \cdot\left|A_{2}-A_{1}\right|}{A_{2}+A_{1}} 100 \% \quad k_{2}=\frac{2 \cdot\left|A_{3}-A_{1}\right|}{A_{1}+A_{3}} 100 \% \quad k_{3}=\frac{2 \cdot\left|A_{2}-A_{3}\right|}{A_{2}+A_{3}} 100 \%
$$


Based on the values of coefficients $\mathrm{k}_{1}, \mathrm{k}_{2}$, and $\mathrm{k}_{3}$, the classification is carried out to assign the selected particles to specific groups, in line with the proposed classification. The assignment algorithm assumes appropriate threshold values for coefficients $\mathrm{k}_{1}, \mathrm{k}_{2}$ and $\mathrm{k}_{3}$. The assignment to the specific groups was based on the criteria listed in Table 1.

a)

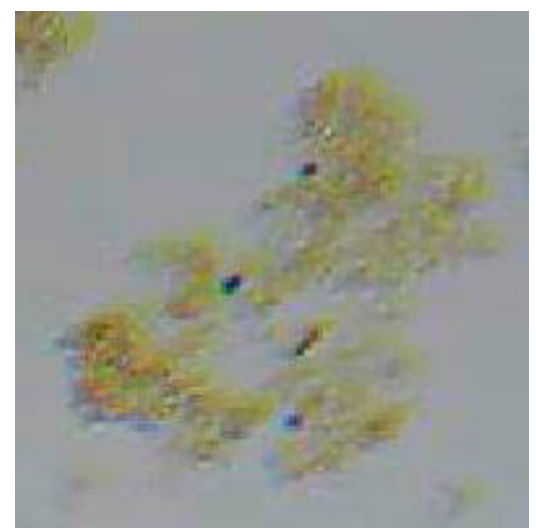

b)

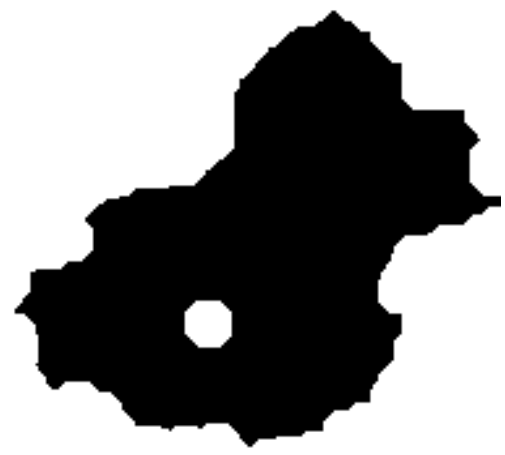

b)

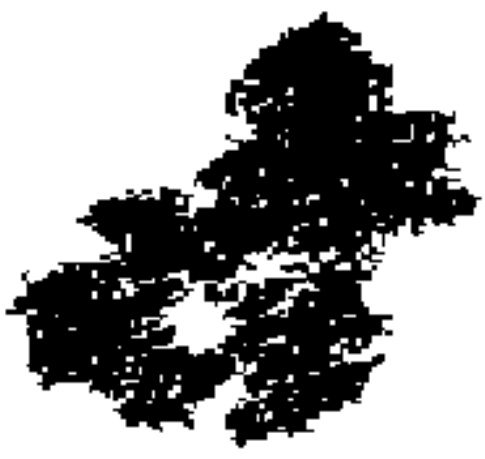

d)

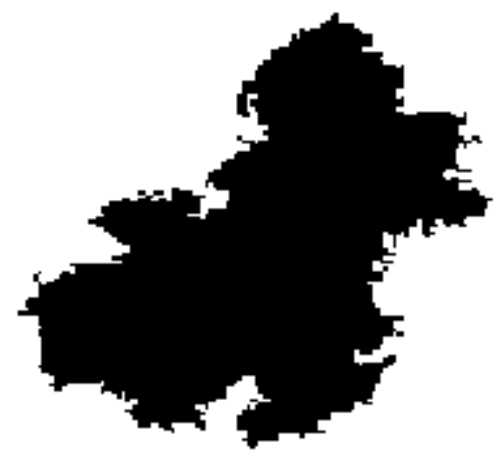

Fig. 5. Image processing: a) microscope image of a particle b) image after binearization c) image after the closure of open spaces d) image after filling in confined spaces.

Table 1. Criterion for the allocation of particles to specific groups.

\begin{tabular}{|c|c|c|c|}
\hline Group name & $\mathbf{k}_{\mathbf{1}}$ & $\mathbf{k}_{\mathbf{2}}$ & $\mathbf{k}_{\mathbf{3}}$ \\
\hline Compact & $\mathrm{k}_{1}<25 \%$ & $\mathrm{k}_{2}<10 \%$ & $\mathrm{k}_{3}<15 \%$ \\
\hline Star-shaped & $\mathrm{k}_{1}>25$ & $\mathrm{k}_{2}<10 \%$ & $\mathrm{k}_{3}>15 \%$ \\
\hline $\begin{array}{c}\text { Porous } \\
\text { conglomerates }\end{array}$ & $\mathrm{k}_{1}>25 \%$ & $\mathrm{k}_{2}>10 \%$ & $\mathrm{k}_{3}>15 \%$ \\
\hline
\end{tabular}

For the purposes of computer classification of particles, it is necessary to determine the appropriate thresholds below which the individual particles are clearly classified. The particles which will be located on the border of threshold numbers are assigned to the group 
for which the results are most similar to the thresholds. In case of not fulfilling one of the three criteria, the particles were classified by meeting as many criteria as possible. The threshold values were determined experimentally.

\section{Experimental studies}

Experimental studies were conducted involving the determination of surface $A_{1}, A_{2}$ and $\mathrm{A}_{3}$ for the particle images (Figures 2, 3 and 4). The results of the calculations are presented in Table 2. The values of the areas were calculated on the basis of the number of pixels comprising the image of the floc. Due to the fact that coefficients $\mathrm{k}_{1}, \mathrm{k}_{2}$, and $\mathrm{k}_{3}$ are the quotients of the areas, it is not necessary to convert the metric units of the surface area.

Table 2. Criterion for the allocation of particles to specific groups.

\begin{tabular}{|c|c|c|c|c|c|c|c|}
\hline $\begin{array}{c}\text { Particle } \\
\text { name }\end{array}$ & $\mathbf{A}_{1}$ & $\mathbf{A}_{2}$ & $\mathbf{A}_{3}$ & $k_{1}[\%]$ & $\mathbf{k}_{2}[\%]$ & $\mathbf{k}_{3}[\%]$ & Group \\
\hline Fig. 2a & 1174 & 1459 & 1336 & 21.65 & $\underline{12.91}$ & 8.80 & compact \\
\hline Fig. $2 b$ & 1851 & 2221 & 1981 & 18.17 & 6.78 & 11.42 & compact \\
\hline Fig. 2c & 1607 & 1999 & 1740 & 21.74 & 7.95 & 13.85 & compact \\
\hline Fig. 3a & 2516 & 3455 & 2721 & 31.45 & 7.83 & 23.77 & Star shape \\
\hline Fig. $3 b$ & 1847 & 2743 & 2052 & 39.04 & 10.52 & 28.82 & Star shape \\
\hline Fig. 3c & 1322 & 1897 & 1388 & 35.73 & 4.87 & 30.99 & Star shape \\
\hline Fig. 4a-1 & 2840 & 3894 & 3268 & 31.30 & 14.01 & 17.48 & $\begin{array}{c}\text { Porous } \\
\text { conglomerates }\end{array}$ \\
\hline Fig. 4a-2 & 2868 & 3948 & 3233 & 31.69 & 11.97 & 19.91 & $\begin{array}{c}\text { Porous } \\
\text { conglomerates }\end{array}$ \\
\hline Fig. $4 \mathrm{~b}$ & 9878 & 14224 & 11340 & 36.06 & 13.78 & 22.56 & $\begin{array}{c}\text { Porous } \\
\text { conglomerates }\end{array}$ \\
\hline
\end{tabular}

During the analysis of the results in Table 2, it was noted that in two cases, for particles with Figure $3 \mathrm{~b}$ and Fig. $2 \mathrm{a}, \quad \mathrm{k}_{2}$ criterion was not fulfilled. This proves the validity of adding third $\mathrm{k}_{3}$ criterion, since in such cases the classification of the particles to a specific group will be met if the other two criteria are fulfilled. This solution improves the reliability of the method of classification.

\section{Summary}

The proposed computer method for the classification of a polydisperse post-coagulation suspension allows the satisfactory classification of its particles. The automated classification method is used for the analysis of microscopic images of such a suspension. This issue is important in view of the fact that studies concerning the settling process that 
contains such suspensions are necessary to learn about the concentration of each fraction suspended in a sedimentation process. In order to apply this method to other suspensions, the appropriate values of coefficients $\mathrm{k}_{1}, \mathrm{k}_{2}$ and $\mathrm{k}_{3}$ must be experimentally determined.

\section{References}

1. R.M. Rząsa, E. Podgórni, Pol. J. Environ. Stud. 23, 6, 2157-2151 (2014)

2. N.D. Tzoupanos, I.A. Zouboulis, 6th IASME/WSEAS International Conference On Heat Transfer, Thermal Engineering And Environment (HTE'08) Rhodes, Greece, August 20-22 (2008)

3. M. Ariffin Abu Hassan, T. Pei Li, Z. Zainon Noor, Journal of Chemical and Natural Resources Engineering, 4, 1, 43-53 (2009)

4. H. Zemmouri, M. Drouiche, A. Sayeh, H. Lounici, N. Mameri, Procedia Engineering 33, 254-260 (2012)

5. R. Bergamasco, L.C. Konradt-Moraes, M.F. Vieira, M.R. Fagundes-Klen, A.M. Salcedo Vieira, Chemical Engineering Journal 2 (2011)

6. Y. Xu, T. Chen, F. Ciu, W. Shi, Chemical Engineering Journal 287, 225-232 (2016)

7. J. Gumińska, M. Kłos, Gaz, woda i technika sanitarna, 5, 195 (2011)

8. M. Świderska-Bróż, J. Krupińska, Ochrona Środowiska, 3, 86, 9-13 (2002)

9. M. Kłos, J. Gumińska, Ochrona Środowiska, 4, 29, 73-76 (2007)

10. G.H. Ganser, Powder Technology, 77, 145 (1993)

11. M. Malakootian, A. Fatehizadeh, Iran. J. Environ. Health. Sci. Eng., 7, 3, 267 (2010)

12. D. Ghernaout, A. Boucherit, Journal of Research \& Developments in Chemistry, $2(2015)$ 